MATHEMATICS OF COMPUTATION

Volume 71, Number 237, Pages 379-391

S 0025-5718(01)01310-2

Article electronically published on March 7, 2001

\title{
SOME BABY-STEP GIANT-STEP ALGORITHMS FOR THE LOW HAMMING WEIGHT DISCRETE LOGARITHM PROBLEM
}

\author{
D. R. STINSON
}

\begin{abstract}
In this paper, we present several baby-step giant-step algorithms for the low hamming weight discrete logarithm problem. In this version of the discrete log problem, we are required to find a discrete logarithm in a finite group of order approximately $2^{m}$, given that the unknown logarithm has a specified number of 1's, say $t$, in its binary representation. Heiman and Odlyzko presented the first algorithms for this problem. Unpublished improvements by Coppersmith include a deterministic algorithm with complexity $O\left(m\left(\begin{array}{c}m / 2 \\ t / 2\end{array}\right)\right)$, and a Las Vegas algorithm with complexity $O\left(\sqrt{t}\left(\begin{array}{c}m / 2 \\ t / 2\end{array}\right)\right)$.

We perform an average-case analysis of Coppersmith's deterministic algorithm. The average-case complexity achieves only a constant factor speed-up over the worst-case. Therefore, we present a generalized version of Coppersmith's algorithm, utilizing a combinatorial set system that we call a splitting system. Using probabilistic methods, we prove a new existence result for these systems that yields a (nonuniform) deterministic algorithm with complexity $O\left(t^{3 / 2}(\log m)\left(\begin{array}{c}m / 2 \\ t / 2\end{array}\right)\right)$. We also present some explicit constructions for splitting systems that make use of perfect hash families.
\end{abstract}

\section{Introduction: the Heiman-Odlyzko algorithm}

Let $G$ be an abelian group, written multiplicatively. Let $\alpha \in G$, and suppose $\beta \in\langle\alpha\rangle$. The discrete logarithm $\log _{\alpha} \beta$ is the unique integer $x$ such that $0 \leq x \leq$ $\operatorname{ord}(\alpha)-1$ and $\alpha^{x}=\beta$. The discrete logarithm problem is to compute $\log _{\alpha} \beta$, given $\alpha$ and $\beta$.

Denote $m=\left\lceil\log _{2}(\operatorname{ord}(\alpha))\right\rceil$. Then the binary representation of $x=\log _{\alpha} \beta$ requires at most $m$ bits, so we can write

$$
x=\sum_{i=0}^{m-1} x_{i} 2^{i},
$$

where $x_{i} \in\{0,1\}$ for $0 \leq i \leq m-1$. The hamming weight of an integer $x$, denoted $\operatorname{wt}(x)$, is the number of 1's in its binary representation.

In cryptographic protocols, such as the Diffie-Hellman key agreement (see, for example, [9]), it is often advantageous to choose an exponent $x$ in such a way that $\alpha^{x}$ can be computed quickly. One way to do this is to choose $x$ to be small relative to $2^{m}$. This scenario is investigated in [10, where the reader is cautioned that a

Received by the editor June 22, 1999 and, in revised form, May 8, 2000.

2000 Mathematics Subject Classification. Primary 68Q25, 11Y16, 05B30.

Key words and phrases. Discrete logarithm problem, baby-step giant-step algorithm, splitting system. 
small choice of $x$ may be insecure. Another slightly more general approach is to choose $x$ such that wt $(x)$ is small compared to $m$. (Given such an $x$, it is faster to compute $\alpha^{x}$ using a typical square-and-multiply algorithm since the number of multiplications is reduced to at most $t+m$, as compared to $3 m / 2$ for a "random" $x$.) However, if wt $(x)$ is too small, then this fact can possibly be exploited by an adversary who is trying to compute $x$. This is the problem we investigate in this paper.

Suppose $t<m$ is a positive integer. Given $\alpha$ and $\beta$, the hamming weight $t$ discrete logarithm problem is to compute $\log _{\alpha} \beta$ whenever $w \mathrm{t}\left(\log _{\alpha} \beta\right)=t$. In this paper, we look at several algorithms for the hamming weight $t$ discrete logarithm problem. The algorithms can be thought of as "baby-step giant-step algorithms" (see, e.g., [9, §3.6.2]).

The binary vector $\left(x_{0}, \ldots, x_{m-1}\right)$ can be regarded as the characteristic vector of a subset of $\mathbb{Z}_{m}$ in an obvious way. This correspondence is made explicit by the two mappings

$$
\text { set }:\left\{0, \ldots, 2^{m}-1\right\} \rightarrow 2^{\mathbb{Z}_{m}}
$$

and

$$
\text { val }: 2^{\mathbb{Z}_{m}} \rightarrow\left\{0, \ldots, 2^{m}-1\right\}
$$

which are defined as

$$
\operatorname{set}(x)=\left\{i: x_{i}=1\right\},
$$

where $\left(x_{0}, \ldots, x_{m-1}\right)$ is the binary representation of $x$, and

$$
\operatorname{val}(Y)=\sum_{i \in Y} 2^{i}
$$

Clearly val and set are inverse functions, and

$$
\operatorname{val}\left(Y_{1} \cup Y_{2}\right)=\operatorname{val}\left(Y_{1}\right)+\operatorname{val}\left(Y_{2}\right)
$$

if $Y_{1} \cap Y_{2}=\emptyset$. It is also clear that wt $(x)=|\operatorname{set}(x)|$ for $0 \leq x \leq 2^{m}-1$.

The following lemmas are easy.

Lemma 1.1. Suppose that $Y_{1}, Y_{2} \subseteq \mathbb{Z}_{m}$ and $\alpha^{\operatorname{val}\left(Y_{1}\right)}=\beta\left(\alpha^{\operatorname{val}\left(Y_{2}\right)}\right)^{-1}$. Then

$$
\log _{\alpha} \beta=\left(\operatorname{val}\left(Y_{1}\right)+\operatorname{val}\left(Y_{2}\right)\right) \bmod \operatorname{ord}(\alpha) \text {. }
$$

Lemma 1.2. Suppose that $\mathrm{wt}\left(\log _{\alpha} \beta\right)=t$, where $t$ is a positive integer. Then there exist subsets $Y_{1}, Y_{2} \subseteq \mathbb{Z}_{m}$ such that $Y_{1} \cap Y_{2}=\emptyset,\left|Y_{1}\right|=\left\lfloor\frac{t}{2}\right\rceil$, and $\alpha^{\operatorname{val}\left(Y_{1}\right)}=$ $\beta\left(\alpha^{\operatorname{val}\left(Y_{2}\right)}\right)^{-1}$.

Lemmas 1.1 and 1.2 are the basis of the following algorithm, independently due to Heiman and Odlyzko [5], which solves the hamming weight $t$ discrete logarithm problem for even $t$.

\section{Algorithm 1.}

1. INPUT: $\alpha, \beta \in G$, an integer $m$ and an even integer $t$

2. For all $Y_{1} \subseteq \mathbb{Z}_{m}$ such that $\left|Y_{1}\right|=t / 2$, compute $\alpha^{\text {val }\left(Y_{1}\right)}$

3. Sort the list of ordered pairs $\left(\operatorname{val}\left(Y_{1}\right), \alpha^{\operatorname{val}\left(Y_{1}\right)}\right)$ by their second coordinates

4. For all $Y_{2} \subseteq \mathbb{Z}_{m}$ such that $\left|Y_{2}\right|=t / 2$, compute $\beta\left(\alpha^{\operatorname{val}\left(Y_{2}\right)}\right)^{-1}$

5 . Sort the list of ordered pairs $\left(\operatorname{val}\left(Y_{2}\right), \beta\left(\alpha^{\operatorname{val}\left(Y_{2}\right)}\right)^{-1}\right)$ by their second coordinates

6. If possible, find $Y_{1}, Y_{2}$ such that $\alpha^{\operatorname{val}\left(Y_{1}\right)}=\beta\left(\alpha^{\operatorname{val}\left(Y_{2}\right)}\right)^{-1}$. 
7. If the previous step is successful, output $\log _{\alpha} \beta=\left(\operatorname{val}\left(Y_{1}\right)+\operatorname{val}\left(Y_{2}\right)\right) \bmod \operatorname{ord}(\alpha)$. Otherwise, output fail.

Remarks. 1. "fail" means that either $\beta \notin\langle\alpha\rangle$ or wt $\left(\log _{\alpha} \beta\right) \neq t$.

2. The complexity of Algorithm (neglecting logarithmic factors) is $O\left(\left(\begin{array}{c}m \\ t / 2\end{array}\right)\right)$. The space requirement is also $O\left(\left(\begin{array}{c}m \\ t / 2\end{array}\right)\right)$.

3. Many variations of the algorithm are possible. For example, it is not necessary to generate the second list and sort it. It suffices to try each $Y_{2}$ and determine if the second coordinate is in the first list by means of a binary search (see, for example, [9, p. 105]).

The remainder of this paper is organized as follows. In Section 2 we describe two algorithms due to Coppersmith. The first algorithm is a deterministic algorithm which we present in terms of new type a combinatorial structure which we call a "splitting system". The second algorithm is a Las Vegas probabilistic algorithm. In Section 3 we perform a detailed average-case analysis of a particular version of the deterministic algorithm. In Section 4 we present several new constructions for splitting systems. For convenience, we assume in Sections 2 through 4 that $m$ and $t$ are both even integers. Then we briefly consider how the algorithms can be modified to handle arbitrary integers $m$ and $t$ in Section 5 Finally, Section 6 is a conclusion.

\section{The Coppersmith Algorithms}

2.1. Splitting families. Coppersmith's algorithm is summarized in [9, p. 128] (it is, in fact, based on an idea from 3]). We describe a generalized version of this algorithm in terms of a type of combinatorial set system that we define now. Suppose $m$ and $t$ are even integers, $0<t<m$. An $(m, t)$-splitting system is a pair $(X, \mathcal{B})$ that satisfies the following properties:

1. $|X|=m$ and $\mathcal{B}$ is a set of $\frac{m}{2}$-subsets of $X$ called blocks;

2. For every $Y \subseteq X$ such that $|Y|=t$, there exists a block $B \in \mathcal{B}$ such that $|B \cap Y|=t / 2$.

We will use the notation $(N ; m, t)$-SS to denote an $(m, t)$-splitting system having $N$ blocks.

Here is a simple construction for splitting systems.

Lemma 2.1 (Coppersmith). For all even integers $m$ and $t$ with $0<t<m$, there exists an $\left(\frac{m}{2} ; m, t\right)-S S$.

Proof. Let $X=\mathbb{Z}_{m}$ and define

$$
B_{i}=\{i+j \bmod m: 0 \leq j \leq m / 2-1\}
$$

for $i \in \mathbb{Z}_{m}$. Let $\mathcal{B}=\left\{B_{i}: 0 \leq i \leq m / 2-1\right\}$. We will show that $(X, \mathcal{B})$ is an $(m, t)$-splitting system.

Fix any subset $Y \subseteq X$ such that $|Y|=t$. For $i \in \mathbb{Z}_{m}$, define

$$
\nu(i)=\left|B_{i} \cap Y\right|-\left|\left(\mathbb{Z}_{m} \backslash B_{i}\right) \cap Y\right| .
$$

If $\nu(0)=0$, then we are done, so assume that $\nu(0) \neq 0$. It is easy to see that $\nu(i)$ is even for all $i, \nu(m / 2)=-\nu(0)$, and $|\nu(i+1)-\nu(i)| \in\{-2,0,2\}$ for all $i$. Therefore there exists some integer $i$ such that $0<i<m / 2$ and $\nu(i)=0$.

Splitting systems can be used to solve the hamming weight $t$ discrete logarithm problem, as follows. 


\section{Algorithm 2.}

1. INPUT: $\alpha, \beta \in G$, even integers $m$ and $t$, and an $(N ; m, t)$-SS, $\left(\mathbb{Z}_{m}, \mathcal{B}\right)$, where $\mathcal{B}=\left\{B_{i}: 0 \leq i \leq N-1\right\}$.

2. For $i=0, \ldots, N-1$, perform the following steps:

(a) For all $Y_{1} \subseteq B_{i}$ such that $\left|Y_{1}\right|=t / 2$, compute $\alpha^{\text {val }\left(Y_{1}\right)}$

(b) Sort the list of ordered pairs $\left(\operatorname{val}\left(Y_{1}\right), \alpha^{\operatorname{val}\left(Y_{1}\right)}\right)$ by their second coordinates

(c) For all $Y_{2} \subseteq \mathbb{Z}_{m} \backslash B_{i}$ such that $\left|Y_{2}\right|=t / 2$, compute $\beta\left(\alpha^{\text {val }\left(Y_{2}\right)}\right)^{-1}$

(d) Sort the list of ordered pairs $\left(\operatorname{val}\left(Y_{2}\right), \beta\left(\alpha^{\operatorname{val}\left(Y_{2}\right)}\right)^{-1}\right)$ by their second coordinates

(e) If possible, find $Y_{1}, Y_{2}$ such that $\alpha^{\operatorname{val}\left(Y_{1}\right)}=\beta\left(\alpha^{\operatorname{val}\left(Y_{2}\right)}\right)^{-1}$.

(f) If the previous step is successful, output $\log _{\alpha} \beta=\operatorname{val}\left(Y_{1}\right)+\operatorname{val}\left(Y_{2}\right) \bmod \operatorname{ord}(\alpha)$ and QUIT. Otherwise, proceed to the next iteration of the FOR loop.

The complexity of Algorithm[2 is $O\left(N\left(\begin{array}{c}m / 2 \\ t / 2\end{array}\right)\right)$. The space requirement is $O\left(\left(\begin{array}{c}m \\ t / 2\end{array}\right)\right)$, which does not depend on $N$. Using the splitting systems described in Lemma 2.1 yields an algorithm having complexity $O\left(m\left(\begin{array}{c}m / 2 \\ t / 2\end{array}\right)\right)$; this is the algorithm that was presented in [9. p. 128].

2.2. A randomized algorithm. A Las Vegas algorithm with good average-case complexity is easy to construct. This algorithm is also due to Coppersmith [2].

\section{Algorithm 3.}

1. INPUT: $\alpha, \beta \in G$, and even integers $m$ and $t$.

2. REPEAT the following steps:

(a) Let $B$ be a random $\frac{m}{2}$-subset of $X$

(b) For all $Y_{1} \subseteq B$ such that $\left|Y_{1}\right|=t / 2$, compute $\alpha^{\text {val }\left(Y_{1}\right)}$

(c) Sort the list of ordered pairs $\left(\operatorname{val}\left(Y_{1}\right), \alpha^{\mathrm{val}\left(Y_{1}\right)}\right)$ by their second coordinates

(d) For all $Y_{2} \subseteq \mathbb{Z}_{m} \backslash B$ such that $\left|Y_{2}\right|=t / 2$, compute $\beta\left(\alpha^{\text {val }\left(Y_{2}\right)}\right)^{-1}$

(e) Sort the list of ordered pairs $\left(\operatorname{val}\left(Y_{2}\right), \beta\left(\alpha^{\operatorname{val}\left(Y_{2}\right)}\right)^{-1}\right)$ by their second coordinates

(f) If possible, find $Y_{1}, Y_{2}$ such that $\alpha^{\operatorname{val}\left(Y_{1}\right)}=\beta\left(\alpha^{\operatorname{val}\left(Y_{2}\right)}\right)^{-1}$.

(g) If the previous step is successful, output $\log _{\alpha} \beta=\operatorname{val}\left(Y_{1}\right)+\operatorname{val}\left(Y_{2}\right) \bmod \operatorname{ord}(\alpha)$ and QUIT. Otherwise, proceed to the next iteration of the REPEAT loop.

The complexity of Algorithm 3 is analyzed as follows. In any iteration, the algorithm is successful if $\left|B \cap \operatorname{set}\left(\log _{\alpha} \beta\right)\right|=t / 2$. This happens with probability

$$
p=\left(\begin{array}{c}
t \\
t / 2
\end{array}\right) \frac{\left(\begin{array}{c}
m-t \\
(m-t) / 2
\end{array}\right)}{\left(\begin{array}{c}
m \\
m / 2
\end{array}\right)} .
$$

We can compute a lower bound on $p$ using the following lemma.

Lemma 2.2 ([7, p. 309]). Suppose that $n$ and $\lambda n$ are positive integers, where $0<$ $\lambda<1$. Define

$$
H(\lambda)=-\lambda \log _{2} \lambda-(1-\lambda) \log _{2}(1-\lambda)
$$

Then

$$
\frac{1}{\sqrt{8 n \lambda(1-\lambda)}} 2^{n H(\lambda)} \leq\left(\begin{array}{c}
n \\
\lambda n
\end{array}\right) \leq \frac{1}{\sqrt{2 \pi n \lambda(1-\lambda)}} 2^{n H(\lambda)} .
$$


Now, applying Lemma 2.2, it is easy to see that

$$
p \geq \sqrt{\frac{\pi}{8}} \sqrt{\frac{m}{t(m-t)}}>c t^{-1 / 2} .
$$

Hence, the complexity of Algorithm 3 is $O\left(\sqrt{t}\left(\begin{array}{c}m / 2 \\ t / 2\end{array}\right)\right)$.

\section{Average-Case analysis of the Deterministic ALGORIthm}

Suppose we use Algorithm 2 with the splitting systems from Lemma 2.1. We consider the average-case complexity of this algorithm, where the average is computed over all $\left(\begin{array}{c}m \\ t\end{array}\right)$ possible exponents having hamming weight $t$. For any integer $x$ with $0 \leq x \leq 2^{m}-1, \operatorname{wt}(x)=t$, let $\psi(x)$ denote the minimum integer $i \geq 0$ such that $\left|B_{i} \cap \operatorname{set}(x)\right|=t / 2$. Then Algorithm 2 requires $\psi(x)+1$ iterations of step 2 if $\beta=\alpha^{x}$. It follows from Lemma 2.1 that $0 \leq \psi(x) \leq m / 2-1$ for all $x$.

Next, define

$$
\delta(m, t)=\sum_{\left\{x: 0 \leq x \leq 2^{m}-1, \mathrm{wt}(x)=t\right\}} \psi(x) .
$$

Then the average-case complexity of the algorithm is in fact $O\left(\left(\frac{\delta(m, t)}{\left(\begin{array}{c}m \\ t\end{array}\right)}+1\right)\left(\begin{array}{c}m / 2 \\ t / 2\end{array}\right)\right)$.

We proceed to develop a formula for $\delta(m, t)$. For any integer $h$ such that $0 \leq$ $h \leq m / 2-1$, we determine the value

$$
\eta(h)=\left|\left\{x: 0 \leq x \leq 2^{m}-1, \operatorname{wt}(x)=t, \psi(x)=h\right\}\right| .
$$

Then it is clear that

$$
\delta(m, t)=\sum_{h=1}^{m / 2-1} h \eta(h) .
$$

First, it is easy to see that

$$
\eta(0)=\left(\begin{array}{c}
m / 2 \\
t / 2
\end{array}\right)^{2}
$$

Next, we have that $\psi(x)=1$ if and only if

$$
\begin{aligned}
x_{0} & =0, \\
|\{1, \ldots, m / 2-1\} \cap \operatorname{set}(x)| & =t / 2-1, \\
x_{m / 2} & =1, \text { and } \\
|\{m / 2+1, \ldots, m-1\} \cap \operatorname{set}(x)| & =t / 2 ;
\end{aligned}
$$

or

$$
\begin{aligned}
x_{0} & =1, \\
|\{1, \ldots, m / 2-1\} \cap \operatorname{set}(x)| & =t / 2, \\
x_{m / 2} & =0, \text { and } \\
|\{m / 2+1, \ldots, m-1\} \cap \operatorname{set}(x)| & =t / 2-1 .
\end{aligned}
$$

From this it follows that

$$
\eta(1)=2\left(\begin{array}{c}
m / 2-1 \\
t / 2-1
\end{array}\right)\left(\begin{array}{c}
m / 2-1 \\
t / 2
\end{array}\right)
$$


Now, let us look at computing $\eta(h)$ for general $h$. Suppose the bit-sequences $\left[x_{0}, \ldots, x_{h-1}\right]$ and $\left[x_{m / 2}, \ldots, x_{m / 2+h-1}\right]$ are fixed. Then it is clearly necessary that the following sum conditions hold for $0 \leq k \leq h-1$ :

$$
\sum_{j=h-1-k}^{h-1} x_{j} \neq \sum_{j=m / 2+h-1-k}^{m / 2+h-1} x_{j}
$$

Denote

$$
s_{1}=\sum_{j=0}^{h-1} x_{j}
$$

and

$$
s_{2}=\sum_{j=m / 2}^{m / 2+h-1} x_{j} .
$$

Then $s_{1} \neq s_{2}$, and $\psi(x)=h$ if and only if (2) holds, and in addition,

$$
|\{h, \ldots, m / 2-1\} \cap \operatorname{set}(x)|=t / 2-s_{1}
$$

and

$$
|\{m / 2+h, \ldots, m-1\} \cap \operatorname{set}(x)|=t / 2-s_{2} .
$$

Let $\zeta\left(h, s_{1}, s_{2}\right)$ denote the number of ways of choosing $x_{0}, \ldots, x_{h-1}$ and $x_{m / 2}, \ldots$, $x_{m / 2+h-1}$ such that the inequality (2) holds for $0 \leq k \leq h-1$. Then, by the discussion above, we have that

$$
\eta(h)=\sum_{s_{1}=0}^{h} \sum_{s_{2}=0}^{h} \zeta\left(h, s_{1}, s_{2}\right)\left(\begin{array}{c}
m / 2-h \\
t / 2-s_{1}
\end{array}\right)\left(\begin{array}{c}
m / 2-h \\
t / 2-s_{2}
\end{array}\right) .
$$

Thus, it remains to find a formula for $\zeta\left(h, s_{1}, s_{2}\right)$. We do this using the familiar "reflection" technique that can be used to determine the well-known formula for the Catalan numbers (see, e.g., [6, §3.4]).

For $0 \leq i \leq h-1$, define $z_{h-i}=x_{i}-x_{m / 2+i}$. Then $z_{i} \in\{0,1,-1\}$ for all $i$. Inequality (2) can then be rewritten as

$$
\sum_{j=1}^{i} z_{j} \neq 0
$$

for $1 \leq i \leq h$.

Given the sequence $\left[z_{1}, \ldots, z_{h}\right]$, we define a path $P=\left[\left(0, y_{0}\right),\left(1, y_{1}\right), \ldots,\left(h, y_{h}\right)\right]$, where $y_{0}=0$ and $y_{i}-y_{i-1}=z_{i}$ for $1 \leq i \leq h$. Observe that $y_{h}=s_{1}-s_{2}$. Also, inequality (3) can be interpreted as saying that the path $P$ never hits the $x$-axis, except for the initial point, $(0,0)$.

For $j_{1}, j_{2} \in\{0,1\}$, define

$$
a_{j_{1}, j_{2}}=\left|\left\{i:\left(x_{i}, x_{m / 2+i}\right)=\left(j_{1}, j_{2}\right)\right\}\right| .
$$


Note that a type $(1,0)$ pair correpsonds to an "up" edge in $P$, a type $(0,1)$ pair correpsonds to a "down" edge in $P$, and type $(0,0)$ and $(1,1)$ pairs correpsond to "horizontal" edges in $P$. We will think of each edge of $P$ as being labelled with an ordered pair in this manner; this will allow the sequences $\left[x_{0}, \ldots, x_{h-1}\right]$ and $\left[x_{m / 2}, \ldots, x_{m / 2+h-1}\right]$ to be recovered from $P$.

It is easy to see that the following equations hold:

$$
\begin{aligned}
a_{0,0}+a_{1,1}+a_{1,0}+a_{0,1} & =h, \\
a_{1,1}+a_{0,1} & =s_{2}, \quad \text { and } \\
a_{1,1}+a_{1,0} & =s_{1} .
\end{aligned}
$$

Then

$$
\left(a_{0,0}, a_{1,1}, a_{1,0}, a_{0,1}\right)=\left(h+j-s_{1}-s_{2}, j, s_{1}-j, s_{2}-j\right)
$$

where $j$ is an integer.

Let us now assume that $s_{1}>s_{2}$ (the case $s_{2}>s_{1}$ can be analyzed in a similar fashion). Then the first edge of $P$ must be labelled $(1,0)$, otherwise (3) will be violated for $i=1$. The total number of such paths $P$ is given by the multinomial coefficient

$$
\left(\begin{array}{c}
h-1 \\
h+j-s_{1}-s_{2}, j, s_{1}-j-1, s_{2}-j
\end{array}\right)
$$

Of course, this total includes paths that do not satisfy (3). Now, suppose that (3) is violated for some $i>1$; let $i_{0}$ be the smallest such $i$. Form a path $P^{*}$ by reflecting the initial portion of $P$ (from $(0,0)$ to $\left.\left(i_{0}, 0\right)\right)$ in the $x$-axis. (Note that a type $(q, r)$ pair becomes a type $(r, q)$ pair after this reflection.)

$P^{*}$ is a path from $(0,0)$ to $\left(h, s_{1}-s_{2}\right)$ in which the initial edge is labelled $(0,1)$. Also, the values $\left(a_{0,0}, a_{1,1}, a_{1,0}, a_{0,1}\right)$ are the same in $P^{*}$ as they are in $P$. The total number of such paths $P^{*}$ is given by the multinomial coefficient

$$
\left(\begin{array}{c}
h-1 \\
h+j-s_{1}-s_{2}, j, s_{1}-j, s_{2}-j-1
\end{array}\right) .
$$

Therefore, it follows that the number of paths $P$ that satisfy all the inequalities (3) is

$$
\left(\begin{array}{c}
h-1 \\
h+j-s_{1}-s_{2}, j, s_{1}-j-1, s_{2}-j
\end{array}\right)-\left(\begin{array}{c}
h-1 \\
h+j-s_{1}-s_{2}, j, s_{1}-j, s_{2}-j-1
\end{array}\right),
$$

which simplifies to give

$$
\frac{s_{1}-s_{2}}{h}\left(\begin{array}{c}
h \\
h+j-s_{1}-s_{2}, j, s_{1}-j, s_{2}-j
\end{array}\right) .
$$

Thus, for $h \neq 0$, it holds that

$$
\zeta\left(h, s_{1}, s_{2}\right)=\frac{\left|s_{1}-s_{2}\right|}{h} \sum_{j=\max \left\{s_{1}+s_{2}-h, 0\right\}}^{\min \left\{s_{1}, s_{2}\right\}}\left(\begin{array}{c}
h \\
h+j-s_{1}-s_{2}, j, s_{1}-j, s_{2}-j
\end{array}\right) .
$$


The sum in (4) can be simplified, as follows:

$$
\begin{aligned}
\sum_{j}\left(\begin{array}{c}
h \\
h+j-s_{1}-s_{2}, j, s_{1}-j, s_{2}-j
\end{array}\right) \\
=\sum_{j}\left(\begin{array}{c}
h-s_{2} \\
s_{1}-j
\end{array}\right)\left(\begin{array}{c}
h-j \\
h-s_{2}
\end{array}\right)\left(\begin{array}{c}
h \\
h-j
\end{array}\right) \\
=\sum_{j}\left(\begin{array}{c}
h-s_{2} \\
s_{1}-j
\end{array}\right)\left(\begin{array}{c}
h \\
s_{2}
\end{array}\right)\left(\begin{array}{c}
s_{2} \\
s_{2}-j
\end{array}\right) \\
=\left(\begin{array}{c}
h \\
s_{2}
\end{array}\right) \sum_{j}\left(\begin{array}{c}
h-s_{2} \\
s_{1}-j
\end{array}\right)\left(\begin{array}{c}
s_{2} \\
j
\end{array}\right) \\
=\left(\begin{array}{c}
h \\
s_{2}
\end{array}\right)\left(\begin{array}{c}
h \\
s_{1}
\end{array}\right) .
\end{aligned}
$$

Combining everything, we get

$$
\begin{aligned}
\delta(m, t)= & \sum_{h=1}^{m / 2-1} \sum_{s_{1}=1}^{m i n\{h, t / 2\}} \sum_{s_{2}=0}^{s_{1}-1} 2\left(s_{1}-s_{2}\right) \\
& \times\left(\begin{array}{c}
m / 2-h \\
t / 2-s_{1}
\end{array}\right)\left(\begin{array}{c}
m / 2-h \\
t / 2-s_{2}
\end{array}\right)\left(\begin{array}{c}
h \\
s_{1}
\end{array}\right)\left(\begin{array}{c}
h \\
s_{2}
\end{array}\right) .
\end{aligned}
$$

We are unable to simplify (5) any further. However, computational evidence show that the speed-up is, at best, only a constant factor. Recalling that the worst-case of the algorithm requires $m / 2$ iterations, the ratio of the average-case to the worst-case complexity is in fact

$$
\frac{\delta(m, t)}{\left(\begin{array}{c}
m \\
t
\end{array}\right)}+\frac{1}{\frac{m}{2}}=\frac{2 \delta(m, t)}{m \frac{m}{t}}+\frac{2}{m} .
$$

Ignoring the term $2 / m$, which tends to 0 as $m \rightarrow \infty$, we compute the ratio

$$
r(m, t)=\frac{2 \delta(m, t)}{m\left(\begin{array}{c}
m \\
t
\end{array}\right)}
$$

for various values of $m$ and $t$. It is clear from the definition of the function $\delta$ that $\delta(m, t)=\delta(m, m-t)$, so it suffices to restrict $t$ so that $2 \leq t \leq m / 2$. We computed all these ratios $r(m, t)$ for even values of $m$ and $t$ such that $2 \leq t \leq m / 2$ and $4 \leq m \leq 80$. We found that the values $r(m, 2)$ decrease as $m$ increases; the values $r(m, m / 2)$ increase as $m$ increases; and, for any fixed value of $m$, the values $r(m, t)$ increase as $t$ increases from 2 to $m / 2$.

Table 1 lists values of $\delta(m, t)$ and $r(m, t)$ for $m \leq 16$ and $t \leq m / 2$; and for $m \in\{24,32,40,48,56,64,72,80\}$ when $t=m / 2$.

It is easy to see from equation (5) that $\delta(m, 2)=\left(m^{3}-4 m\right) / 24$. Hence $r(m, 2) \rightarrow$ $1 / 6$ as $m \rightarrow \infty$. The numerical evidence also suggests strongly that $r(m, m / 2)=$ $m /(4 m+8)$ for $m \equiv 0 \bmod 4$. We verified that this is indeed the case for $m \leq 100$, though we do not know how to prove it in general. 
TABLE 1.

\begin{tabular}{rrrr}
$m$ & $t$ & $\delta(m, t)$ & $r(m, t)$ \\
\hline 4 & 2 & 2 & .166667 \\
6 & 2 & 8 & .177778 \\
8 & 2 & 20 & .178571 \\
8 & 4 & 56 & .200000 \\
10 & 2 & 40 & .177778 \\
10 & 4 & 216 & .205714 \\
12 & 2 & 70 & .176768 \\
12 & 4 & 616 & .207407 \\
12 & 6 & 1188 & .214286 \\
14 & 2 & 112 & .175824 \\
14 & 4 & 1456 & .207792 \\
14 & 6 & 4576 & .217687 \\
16 & 2 & 168 & .175000 \\
16 & 4 & 3024 & .207692 \\
16 & 6 & 14040 & .219156 \\
16 & 8 & 22880 & .222222 \\
24 & 12 & 7488432 & .230769 \\
32 & 16 & 2262890880 & .235294 \\
40 & 20 & 656412042000 & .238095 \\
48 & 24 & 185746197214656 & .240000 \\
56 & 28 & 51694598543070560 & .241379 \\
64 & 32 & 14216720608524338688 & .242424 \\
72 & 36 & 3874974677018786931408 & .243243 \\
80 & 40 & 1048850816910596843528000 & .243902
\end{tabular}

\section{IMPROVED RESULTS CONCERNING SPLITTING SYSTEMS}

4.1. Probabilistic methods. We can improve Algorithm2 by constructing smaller splitting systems. We first provide a bound using probabilistic methods. Let $m$ and $t$ be even integers such that $0<t<m$. Suppose that $\mathcal{B}$ a set of $\frac{m}{2}$-subsets of an $m$-set, $X$, and $|\mathcal{B}|=N$. For a subset $Y \subseteq X$ with $|Y|=t$, define $Z_{Y}(\mathcal{B})=0$ if there exists a block $B \in \mathcal{B}$ such that $|B \cap Y|=t / 2$, and define $Z_{Y}(\mathcal{B})=1$, otherwise. Let $Z_{Y}$ denote the random variable obtained by letting $\mathcal{B}$ be a set of $N$ randomly chosen $\frac{m}{2}$-subsets of $X$. Clearly, the expected value of $Z_{Y}$, denoted $E\left[Z_{Y}\right]$, is $(1-p)^{N}$, where

$$
p=\frac{\left(\begin{array}{c}
t \\
t / 2
\end{array}\right)\left(\begin{array}{c}
m-t \\
m-t) / 2
\end{array}\right)}{\left(\begin{array}{c}
m \\
m / 2
\end{array}\right)} .
$$

If we define the random variable

$$
Z=\sum_{\{Y \subseteq X:|Y|=t\}} Z_{Y}
$$


then we have $E[Z]=\left(\begin{array}{c}m \\ t\end{array}\right)(1-p)^{N}$. It is clear that there exists an $(N ; m, t)$-SS if $E[Z]<1$. Since $\left(\begin{array}{c}m \\ t\end{array}\right)<m^{t}$, this will be true if

$$
t \log m+N \log (1-p)<0,
$$

which is equivalent to

$$
N>\frac{t \log m}{-\log (1-p)}
$$

Using elementary calculus, we have that $-\log (1-p)>p$; and $p \geq c t^{-1 / 2}$ was shown in equation (11). Hence, an $(N ; m, t)$-SS exists if

$$
N>\frac{1}{c} t^{3 / 2} \log m
$$

Thus we have proven the following result.

Theorem 4.1. For any even integers $t$ and $m$ with $0<t<m$, there exists an $(N ; m, t)$-SS with $N \approx c_{0} t^{3 / 2} \log _{2} m$, where $c_{0}$ is a constant.

Thus, Theorem 4.1 yields a (nonuniform) deterministic algorithm having complexity $O\left(t^{3 / 2}(\log m)\left(\begin{array}{c}m / 2 \\ t / 2\end{array}\right)\right)$.

4.2. Explicit constructions. In this section, we present a recursive construction for splitting families that uses perfect hash families. Perfect hash families were introduced by Mehlhorn (see, e.g., [8]) and have been studied extensively since then (for a recent survey, see [4]).

We require some definitions. Let $n \geq m$ be positive integers. An $(n, m)$-hash function is a function $h: A \rightarrow B$, where $|A|=n$ and $|B|=m$. The hash function $h$ is said to be balanced provided that $\left|h^{-1}(y)\right|=n / m$ for all $y \in B$.

Let $n, m$ and $w$ be integers such that $n \geq m \geq w \geq 2$. An $(n, m, w)$-perfect hash family is a finite set $\mathcal{H}$ of $(n, m)$-hash functions such that $h: A \rightarrow B$ for each $h \in \mathcal{H}$, where $|A|=n$ and $|B|=m$, with the property that for any $X \subseteq A$ with $|X|=w$, there exists at least one $h \in \mathcal{H}$ such that $\left.h\right|_{X}$ is one-to-one. $\mathcal{H}$ is said to be an $(n, m, w)$-balanced perfect hash family if $\mathcal{H}$ is an $(n, m, w)$-perfect hash family and $h$ is balanced for every $h \in \mathcal{H}$.

We will use the notation $\operatorname{BPHF}(N ; n, m, w)$ to denote an $(n, m, w)$-balanced perfect hash family with $|\mathcal{H}|=N$. We can depict a $\operatorname{BPHF}(N ; n, m, w)$ as an $N \times n$ array on $m$ symbols, say $A$, where each row of $A$ corresponds to one of the functions in the family. This array has the property that, for any subset of $w$ columns, there exists at least one row such that the entries in the $w$ given columns of that row are distinct; and any row of $A$ contains exactly $n / m$ occurrences of each symbol.

Here is a recursive construction for splitting families.

Theorem 4.2. Suppose there exist a $\operatorname{BPHF}\left(N_{0} ; n, m, t\right)$ and an $S S\left(N_{1} ; m, t\right)$. Then there exists an $S S\left(N_{0} N_{1} ; n, t\right)$.

Proof. Let $M$ be the $N_{1} \times m$ incidence matrix of an $\operatorname{SS}\left(N_{1} ; m, t\right)$, and denote the columns of $M$ by $c_{1}, \ldots, c_{m}$. Let $A$ be the array representation of the $\operatorname{BPHF}\left(N_{0} ; n, m, t\right)$, and replace each entry $y=A(i, j)$ by the column vector $c_{y}$. Call the resulting matrix $M_{1}$.

It is easy to see that $M_{1}$ is the incidence matrix of an $\operatorname{SS}\left(N_{0} N_{1} ; n, t\right)$ : The "balance" property of the hash family ensures that each block of the resulting splitting system has cardinality $n / 2$. Also, given a $t$-subset of points, say $B_{1}$, 
there exists a hash function $h$ such that $\left.h\right|_{B_{1}}$ is injective. Restricting to the $N_{1}$ corresponding rows of $M_{1}$, property 2 of splitting families is inherited from $M$. 4.2 .

The following corollary is an immediate application of Lemma 2.1 and Theorem

Corollary 4.3. If there exists a $\operatorname{BPHF}\left(N_{0} ; n, m, t\right)$, then there exists an $S S\left(N_{0} m / 2 ; n, t\right)$.

In order to apply Theorem 4.2 or Corollary 4.3, we need balanced perfect hash families. It is not difficult to verify that certain direct constructions for perfect hash families in the literature yield BPHF. We illustrate with an example.

Let $q$ be a prime power. An $(N, K, D, q)$-code is a set $\mathcal{C}$ of $K$ vectors in $\left(\mathbb{F}_{q}\right)^{N}$ such that the Hamming distance between any two distinct vectors in $\mathcal{C}$ is at least $D$. The code $\mathcal{C}$ is linear if it is a subspace of $\left(\mathbb{F}_{q}\right)^{N}$; in this case $K=q^{k}$, where $k=\operatorname{dim}(\mathcal{C})$.

Theorem 4.4. If a linear $(N, K, D, q)$-code exists, then there exists a $B P H F(N ; K, q, w)$, provided that

$$
\frac{D}{N}>1-\frac{1}{\left(\begin{array}{c}
w \\
2
\end{array}\right)} .
$$

Proof. Construct an $N \times K$ array whose columns are the codewords in $\mathcal{C}$. It is shown in [1] that this array is a $\operatorname{PHF}(N ; K, q, w)$ provided that $D / N>1-\left(1 /\left(\begin{array}{c}w \\ 2\end{array}\right)\right)$. Since $\mathcal{C}$ is linear, it follows that each hash function in the family is balanced, and the result follows.

Using Reed-Solomon codes, we obtain the following corollary of Theorem 4.4.

Corollary 4.5. Suppose that $q$ is a prime power, $0<\ell<q$ is an integer, and $q>(\ell-1)\left(\begin{array}{c}w \\ 2\end{array}\right)$. Then there exists a $\operatorname{BPHF}\left(q ; q^{\ell}, q, w\right)$.

Proof. A $q$-ary dimension $\ell$ extended Reed-Solomon code of length $q$ exists (see, for example, [7]). This is a linear $\left(q, q^{\ell}, q-\ell+1, q\right)$-code. Apply Theorem 4.4.

Combining Corollaries 4.3 and 4.5 allows us to prove an interesting asymptotic existence theorem. Suppose $m$ and $t$ are given, and we want to construct an $\operatorname{SS}(N ; m, t)$. Choose $q \approx t^{2} \log m$ and $\ell \approx \log m / \log q$. Then all necessary conditions are satisfied, and we obtain an $\operatorname{SS}(N ; m, t)$ in which $N$ is $O\left(t^{4}(\log m)^{2}\right)$.

\section{AlgorithmS FOR ARBItraRy $m$ AND $t$}

In this section, we discuss briefly how the algorithms we have presented can be modified to handle the cases where one or both of $m$ and $t$ are odd. First, Algorithm 11does not care if $m$ is even or odd. So we only need to consider the case when $t$ is odd. In this case, it suffices to consider all $Y_{1} \subseteq \mathbb{Z}_{m}$ with $\left|Y_{1}\right|=\left\lfloor\frac{t}{2}\right\rfloor$ in step 3; and all $Y_{2} \subseteq \mathbb{Z}_{m}$ with $\left|Y_{2}\right|=\left\lceil\frac{t}{2}\right\rceil$ in step 4 .

Algorithm $[3$ is also easy to modify. In step 2(a), $B$ should be chosen to be a random $\left\lfloor\frac{m}{2}\right\rfloor$-subset; in step 2(b) consider all $Y_{1} \subseteq B$ with $\left|Y_{1}\right|=\left\lfloor\frac{t}{2}\right\rfloor$; and in step 2(d) consider all $Y_{2} \subseteq \mathbb{Z}_{m} \backslash B$ with $\left|Y_{2}\right|=\left\lceil\frac{t}{2}\right\rceil$. The complexity analyses of the modified versions of Algorithm 1 and 3 are straightforward.

Now we proceed to Algorithm 2 We defined splitting systems in Section 2.1 assuming that $m$ and $t$ are both even. We now generalize this definition to arbitrary 
integers $m$ and $t$ with $0<t<m$. An $(m, t)$-generalized splitting system is a pair $(X, \mathcal{B})$ that satisfies the following properties:

1. $|X|=m$ and $\mathcal{B}$ is a set of $\left\lfloor\frac{m}{2}\right\rfloor$-subsets of $X$ called blocks;

2. For every $Y \subseteq X$ such that $|Y|=t$, there exists a block $B \in \mathcal{B}$ such that $|B \cap Y|=\left\lfloor\frac{t}{2}\right\rfloor$.

We will use the notation $(N ; m, t)$-GSS to denote an $(m, t)$-generalized splitting system having $N$ blocks.

Given an $(N ; m, t)$-GSS, we can apply Algorithm 2 with the following modifications: in step 2(a) consider all $Y_{1} \subseteq B_{i}$ with $\left|Y_{1}\right|=\left\lfloor\frac{t}{2}\right\rfloor$; and in step 2(c) consider all $Y_{2} \subseteq \mathbb{Z}_{m} \backslash B_{i}$ with $\left|Y_{2}\right|=\left\lceil\frac{t}{2}\right\rceil$.

When $m$ and $t$ are both even, it is clear that an $(N ; m, t)$-GSS is the same thing as an $(N ; m, t)$-SS, and Algorithm 2 is not changed. The following constructions for generalized splitting systems allow us to handle the cases where at least one of $m$ and $t$ is odd.

Theorem 5.1. Suppose that $m$ and $t$ are even and there exists an $(N ; m, t)-S S$. Then the following exist: a $(2 N ; m, t-1)-G S S$; an $(N ; m-1, t)-G S S$; and an $(N ; m-1, t-1)-G S S$.

Proof. Let $(X, \mathcal{B})$ be an $(N ; m, t)$-SS, where $m$ and $t$ are both even. First we show that $(X, \mathcal{B} \cup\{X \backslash B: B \in \mathcal{B}\})$ is a $(2 N ; m, t-1)$-GSS. Suppose that $Y \subseteq X$, $|Y|=t-1$. Let $x \in X \backslash Y$, and define $Y^{\prime}=Y \cup\{x\}$. Since $|Y|=t$, there exists a block $B \in \mathcal{B}$ such that $\left|Y^{\prime} \cap B\right|=t / 2$. If $x \in B$, then

$$
|Y \cap B|=\frac{t}{2}-1=\left\lfloor\frac{t-1}{2}\right\rfloor .
$$

Otherwise, $x \in X \backslash B$, in which case

$$
|Y \cap(X \backslash B)|=\frac{t}{2}-1=\left\lfloor\frac{t-1}{2}\right\rfloor .
$$

Therefore we have constructed a $(2 N ; m, t-1)$-GSS.

Now we construct an $(N ; m-1, t)$-GSS and an $(N ; m-1, t-1)$-GSS. Pick an arbitrary point $x_{0} \in X$. We can assume without loss of generality that $x_{0} \in B$ for all $B \in \mathcal{B}$ (for each $B$ such that $x_{0} \notin B$, replace $B$ by $X \backslash B$ ).

Define $X^{\prime}=X \backslash\left\{x_{0}\right\}$, and for all $B \in \mathcal{B}$, define $B^{\prime}=B \backslash\left\{x_{0}\right\}$. Let $\mathcal{B}^{\prime}=$ $\left\{B^{\prime}: B \in \mathcal{B}\right\}$. We will show that $\left(X^{\prime}, \mathcal{B}^{\prime}\right)$ is simultaneously an $(N ; m-1, t)$-GSS and an $(N ; m-1, t-1)$-GSS.

First, suppose $Y^{\prime} \subseteq X^{\prime},\left|Y^{\prime}\right|=t$. There exists a block $B \in \mathcal{B}$ such that $\left|Y^{\prime} \cap B\right|=t / 2$. Then $\left|Y^{\prime} \cap B^{\prime}\right|=t / 2$, as desired. Therefore we have constructed an $(N ; m-1, t)$-GSS.

Finally, suppose that $Y^{\prime} \subseteq X^{\prime},\left|Y^{\prime}\right|=t-1$. Define $Y=Y^{\prime} \cup\left\{x_{0}\right\}$. There exists a block $B \in \mathcal{B}$ such that $|Y \cap B|=t / 2$. Then

$$
\left|Y^{\prime} \cap B^{\prime}\right|=\frac{t}{2}-1=\left\lfloor\frac{t-1}{2}\right\rfloor
$$

as desired. Therefore, we have constructed an $(N ; m-1, t-1)$-GSS. 


\section{Conclusion}

We have described several varaints of baby-step giant-step algorithms for the low hamming weight discrete logarithm problem. For practical use, Coppersmith's Las Vegas algorithm (Algorithm 3) would be preferred. If a deteministic algorithm is desired, then an algorithm based on the idea of splitting systems can be used. This is a generalization of another algorithm due to Coppersmith. We performed an average case analysis of the simplest of these algorithms and found that only a constant factor speedup is acheived, as compared to the worst case. Several alternative methods of constructing splitting systems were investigated. These permit construction of smaller splitting systems, and hence more efficient determinstic algorithms, at least asymptotically. Finding more efficient methods of constructing splitting systems is an interesting combinatorial problem.

\section{ACKNOWLEDGMENTS}

The author's research is supported by the Natural Sciences and Engineering Research Council of Canada through the following grants: NSERC-IRC \#21643196 and NSERC-RGPIN \#203114-98.

I would like to thank Ruizhong Wei for his help with computations and for his useful comments; Alfred Menezes for his assistance with references; and the referees for many helpful suggestions concerning the presentation of the results in this paper.

\section{REFERENCES}

[1] N. Alon. Explicit construction of exponential sized families of $k$-independent sets, Discrete Math. 58 (1986), 191-193. MR 87e:05002

[2] D. Coppersmith. Private communication to Scott Vanstone, December 1997.

[3] D. Coppersmith and G. Seroussi. On the minimum distance of some quadratic residue codes, IEEE Trans. Inform. Theory 30 (1984), 407-411. MR 86c:94025

[4] Z. J. Czech, G. Havas and B. S. Majewski. Perfect hashing, Theoretical Computer Science 182 (1997), 1-143. MR 98h:68048

[5] R. Heiman. A note on discrete logarithms with special structure. Lecture Notes in Computer Science 658, 454-457 (Advances in Cryptology - EUROCRYPT '92).

[6] D. L. Kreher and D. R. Stinson. Combinatorial algorithms: generation, enumeration and search, CRC Press, 1999.

[7] F. J. MacWilliams and N. J. A. Sloane. The theory of error-correcting codes, North-Holland, 1977. MR 57:5408a MR 57:5408b

[8] K. Mehlhorn. Data structures and algorithms 1: sorting and searching, Springer-Verlag, Berlin, 1984. MR 86e:68001

[9] A. J. Menezes, P. C. van Oorschot and S. A. Vanstone. Handbook of applied cryptography, CRC Press, 1997. MR 99g:94015

[10] P. C. van Oorschot and M. J. Wiener. On Diffie-Hellman key agreement with short exponents. Lecture Notes in Computer Science 1070, 332-343 (Advances in Cryptology EUROCRYPT '96), Springer, Berlin, 1996. CMP 97:04

Department of Combinatorics and Optimization, University of Waterloo, Waterloo Ontario, N2L 3G1, Canada

E-mail address: dstinson@uwaterloo.ca 